\title{
HI Absorption Measurements over the Galactic Centre Radio Arc and Arches Regions
}

\author{
J. Lasenby and A.N. Lasenby \\ Mullard Radio Astronomy Observatory, Cambridge, UK. \\ F. Yusef-Zadeh ${ }^{1}$ \\ NASA-Goddard Space Flight Center and Northwestern University
}

\begin{abstract}
An HI absorption study of the central $\sim 100 \mathrm{pc}$ of the galactic centre was performed using the compact array of the VLA ${ }^{2}$. The results of these observations lead us to believe that the body of gas at $40-50 \mathrm{~km} \mathrm{~s}^{-1}$, known as the ' $40 \mathrm{~km} \mathrm{~s}^{-1}$ molecular cloud', is placed behind the Arc. In addition, the distribution of gas around $+20 \mathrm{~km} \mathrm{~s}^{-1}$ across the Arc and its correlation with $3 \mathrm{~cm}$ polarized emission suggests that it may be this $20 \mathrm{~km} \mathrm{~s}^{-1}$ gas which is associated with the Arc and is depolarizing much of its synchrotron radiation. The kinematic structure of the negative velocity $\mathrm{HI}$ gas across the arched filaments implies an association between the neutral atomic gas and the ionized and molecular material.
\end{abstract}

\section{Introduction}

The HI absorption study described here is directed towards the question of the relative positionings and possible physical associations of the major features in the galactic centre region. Amongst the features covered are the Radio Arc - the system of narrow, synchrotron-emitting filamentary structures aligned almost perpendicular to the galactic plane at $l \approx 0.18^{\circ}$ - and the series of curved structures, known as the Arches, which appear to connect the Arc to the Sgr A halo. (The results for the Sgr A region itself are covered in a separate contribution.) Of major interest was the relation of the continuum features to the large molecular condensations in the region. Absorption studies provide an ideal way of establishing this, but previous studies in HI have only been carried out at higher resolutions, covering the Sgr A complex alone. The present observations are designed to be complementary to these studies, having sensitivity to lower brightness, larger scale structure rather than point sources, and to relate to angular scales similar to those utilized in many $\mathrm{CO}$ studies. One of the key questions to be addressed concerns the placement of the Radio Arc with respect to the galactic centre and to the ' $40 \mathrm{~km} \mathrm{~s}^{-1}$ molecular cloud'. Yusef-7adeh et al. 1984, have outlined several arguments supporting the claim that the filaments of the Radio Arc are related to the galactic nucleus, and one of the most convincing of these involves the appearance of the $40 \mathrm{~km} \mathrm{~s}^{-1}$ cloud in $\mathrm{NH}_{3}(1,1)$ and ${ }^{13} \mathrm{CO}$ emission. $\mathrm{NH}_{3}(1,1)$ emission contours at $40 \mathrm{~km} \mathrm{~s}^{-1}$ (Güsten et al. 1981) appear to trace the outline of G0.18-0.04, a radio structure often called the 'sickle', seen where the filaments cross the galactic plane, while the main body of the ${ }^{13} \mathrm{CO}$ gas between +40 and $+50 \mathrm{~km} \mathrm{~s}^{-1}$ seems to lie at the edge of the Radio Arc (Bally et al. 1987). The sickle feature is also the only region to display significant radio recombination line emission around $+40 \mathrm{~km} \mathrm{~s}^{-1}$ (Pauls

${ }^{1}$ National Research Council Resident Research Associate

${ }^{2}$ The VLA is part of the National Radio Astronomy Observatory under contract to the NSF. 


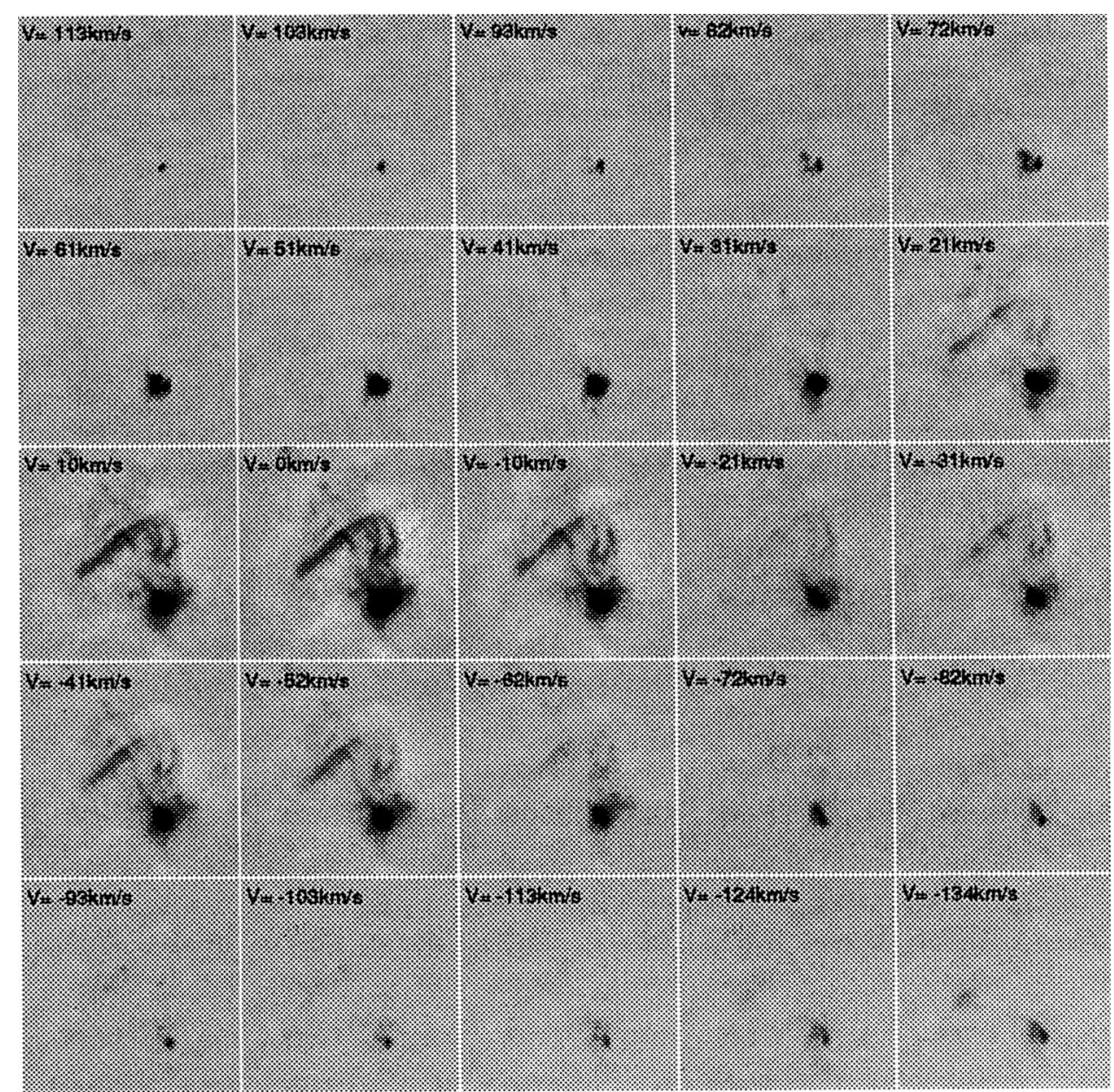

Figure 1: HI absorption line-minus-continuum (difference) maps for channels 21 to 45 . The central velocity of the channel is shown in the top left hand corner of each frame. In every channel map the negative log of the absolute value of the difference (in Jy/beam) has been taken, and the greyscale range is 0 (white) to -0.5 (black) in all cases. Each frame covers the same area as shown Figure 3 . The maps have not been corrected for primary beam attenuation. 
and Mezger, 1980; Yusef-Zadeh et al. 1987). Since there is a good case for sickle-filament interaction (Yusef-Zadeh, 1986 [hereafter YZ86]), any interaction of the sickle with the $40 \mathrm{~km} \mathrm{~s}^{-1}$ cloud would then place the Arc at the galactic centre. We shall however see that, contrary to previous expectation, the $40 \mathrm{~km} \mathrm{~s}^{-1}$ cloud displays no absorption over the Radio Arc at all. Instead, the HI results suggest that material at $+20 \mathrm{~km} \mathrm{~s}^{-1}$ appears to be more closely connected with the Arc. (We can however limit the Arc to within $\sim 200 \mathrm{pc}$ of the galactic centre.) Also considered is the association between neutral, ionized and molecular material at negative velocities over the Arch region.

\section{Observations and Results}

The HI absorption observations of the galactic centre region to be described here were carried out using the VLA C/D hybrid array. A detailed description of the observations is given elsewhere (Lasenby et al. 1988), but here we briefly report the results for HI absorption toward the Radio Arc and Arches. The maps presented are uniformly weighted and have $53^{\prime \prime} \times 45^{\prime \prime}$ spatial and $\sim 10.2 \mathrm{~km} \mathrm{~s}^{-1}$ velocity resolution. The bandwidth of $3.125 \mathrm{MHz}$ gave a total coverage of about $660 \mathrm{~km} \mathrm{~s}^{-1}$. The optical depth maps were constructed using a new statistical technique (see, Lasenby et al. 1988) such that for each point on the map one can be $\sim 95 \%$ confident that the fractional error in the optical depth value is $\lesssim \mathbf{2 0 \%}$. The resulting maps are therefore more accurate and reliable than those made by standard methods. The uniformly weighted line-minus-continuum maps for channels 21 to 45 are shown in Figure 1. The numbers in the top left hand corner of each map show the central velocity of the channel. It should be noted here that the maps of Figure 1 have not been corrected for primary beam attenuation. This will not, however, affect the calculation of quantities, such as optical depth, formed from the division of two maps. We now discuss some of the features occurring across the range of observed velocities.

+40 to $50 \mathrm{~km} \mathrm{~s}^{-1}$ Material: Figure 2 shows maps of optical depth at +40 and $+50 \mathrm{~km} \mathrm{~s}^{-1}$ and it is apparent from these, and from Figure 1, that there is no evidence of absorption against the Radio Arc or Arches at these velocities (both maps cover the same region as shown in Figure 3). The gas in front of the Sgr A complex is, however, well traced in Figure 2. Thus, either the Arc lies in front of the $40 \mathrm{~km} \mathrm{~s}^{-1}$ cloud, or the Arc lies behind (or in the vicinity), but the column density of the cloud drops off abruptly as the western end of the Arc is approached. One can estimate an $\mathrm{H}_{2}$ column density across the Arc region of $\mathrm{N}\left(\mathrm{H}_{2}\right) \simeq(1.1$ to 4.8$) \times 10^{22} \mathrm{~cm}^{-2}$ per $10 \mathrm{~km} \mathrm{~s}^{-1}$ bin for the 40 to $60 \mathrm{~km} \mathrm{~s}^{-1}$ material: this is based on ${ }^{12} \mathrm{CO}$ and ${ }^{13} \mathrm{CO}$ data from Liszt et al. 1985 and Bally et al. 1987 respectively. Such a column density would imply that in the direction of the Arc we should expect to see $\tau \gtrsim 0.6 \mathrm{~km} \mathrm{~s}^{-1}$ (over a $10 \mathrm{~km} \mathrm{~s}^{-1}$ bin). Figure 2 shows that no $\tau$ is observed greater than $\sim 0.05 \mathrm{~km} \mathrm{~s}^{-1}$. The above calculations are, of course, affected by the uncertainties in quantities such as the $\mathrm{H}_{2}:{ }^{13} \mathrm{CO}$ abundance ratio, $T_{\text {spin }}(\mathrm{HI})$ etc. However, if one makes similar analyses on other bodies of gas which do absorb the Arc - e.g., the $-50 \mathrm{~km} \mathrm{~s}^{-1}$ material or the $-130 \mathrm{~km} \mathrm{~s}^{-1}$ gas - it is found that the $\tau$ maps do show contours across the Arc at the expected levels. This fact therefore gives us confidence in concluding that the $40 \mathrm{~km} \mathrm{~s}^{-1}$ cloud lies wholly behind the Radio Arc. Thus, while on the one hand there appears to be good evidence for $40 \mathrm{~km} \mathrm{~s}^{-1}$ cloud - Arc interaction, as witnessed by the 


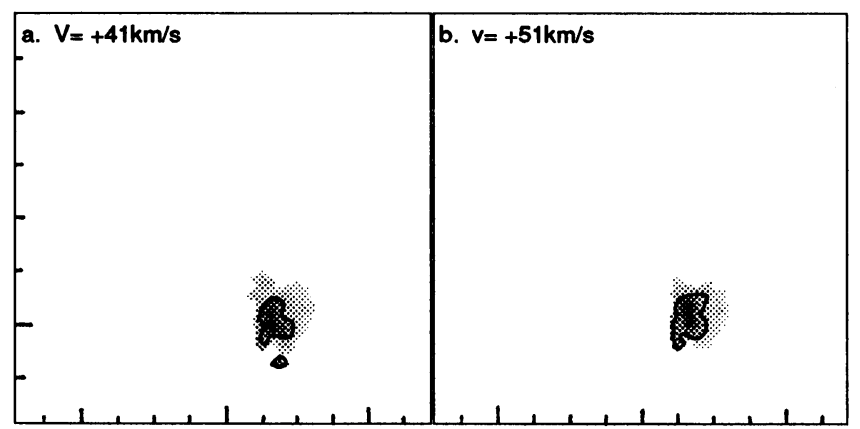

Figure 2: Greyscale maps of $\mathrm{HI}$ optical depth centred on, a) $\sim+41 \mathrm{~km} \mathrm{~s}^{-1}$ and b) $\sim+52 \mathrm{~km} \mathrm{~s}^{-1}$. In both cases the map scales are linear, with $\int_{\Delta v \simeq 10} \tau d v$ ranging from 0 (white) to $1.78 \mathrm{~km} \mathrm{~s}^{-1}$ (black) in a), and from 0 (white) to $1.83 \mathrm{~km} \mathrm{~s}^{-1}$ (black) in b). The contours on each map show the $\int_{\Delta v \simeq 10} \tau d v=$ 0.5 level.

Figure 3: Greyscale map of HI optical depth for channel 30 , centred on $\sim+21 \mathrm{~km} \mathrm{~s}^{-1}$. Map scale is linear, with $\int_{\Delta v \simeq 10} \tau d v$ ranging from 0 to $2.67 \mathrm{~km} \mathrm{~s}^{-1}$. Note the apparent 'gap' in the $20 \mathrm{~km} \mathrm{~s}^{-1}$ material across the central section of the Arc. The solid contours show $3 \mathrm{~cm}$ polarized continuum emission taken from Seiradakis et al. 1985, with A marking the peak of this emission.

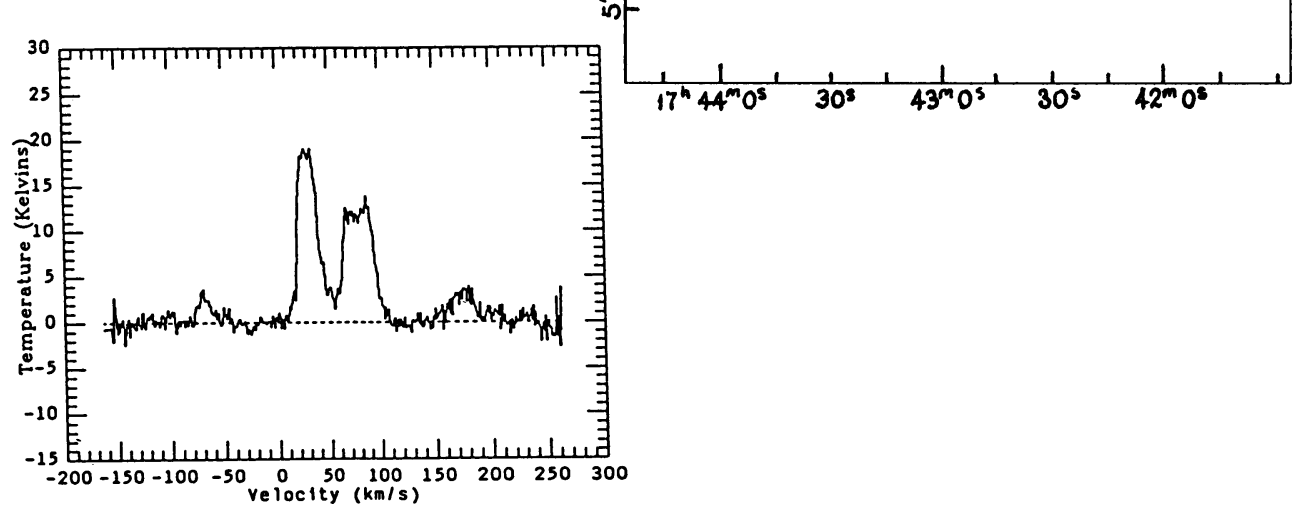

Figure 4: ${ }^{12} \mathrm{CO} \mathrm{J}=2 \rightarrow 1$ spectrum taken at $\alpha=17^{h} 43^{m} 9.6^{\circ}, \delta=-28^{\circ} 47^{\prime} 8.0^{\prime \prime}$, lying at a point in the Arc at which the HI optical depth at $\sim 20 \mathrm{~km} \mathrm{~s}^{-1}$ is large. The temperature scale is $T_{A}^{*}$. The spectrum was kindly taken for us during commissioning of the JCMT by R. Padman \& N. Parker. 
$\mathrm{NH}_{3}$ and ${ }^{13} \mathrm{CO}$ emission (Güsten et al. 1981; Bally et al. 1987) and the recombination line studies (Pauls and Mezger, 1980) described earlier, on the other hand, the HI absorption measurements give no indication of such an interaction. The lack of absorption at 40 to $50 \mathrm{~km} \mathrm{~s}^{-1}$ cannot, of course, wholly rule out the situation in which the $40 \mathrm{~km} \mathrm{~s}^{-1}$ cloud lies immediately behind the Arc but does interact with the Arc in sorne way.

$+20 \mathrm{kms}^{-1}$ Material: The optical depth map at $+20 \mathrm{~km} \mathrm{~s}^{-1}$ of Figure 3 shows that while the $20 \mathrm{~km} \mathrm{~s}^{-1}$ material apparently covers the northern and southern tips of the Arc, there is a central gap just below G0.18-0.04 where there is little or no gas. The ${ }^{13} \mathrm{CO} \mathrm{J}=1 \rightarrow 0$ map at $20-30 \mathrm{~km} \mathrm{~s}^{-1}$ of Bally et al. (1987) shows similarly placed condensations. It is also interesting to note that the single dish recombination line study of Pauls and Mezger (1980), showed emission at $\sim+20 \mathrm{~km} \mathrm{~s}^{-1}$ covering the sickle and northern Arc and apparently centred eastwards of the Arc. This might therefore lead us to believe that the neutral gas, as seen in HI absorption, the molecular gas traced by ${ }^{13} \mathrm{CO}$ (Bally et al. 1987) and the ionized material (Pauls and Mezger, 1980) at $\sim+20 \mathrm{~km} \mathrm{~s}^{-1}$ are physically related. In addition to this, there is also evidence of $\mathrm{H} 110 \alpha$ recombination line emission at $20-30 \mathrm{~km} \mathrm{~s}^{-1}$ over the sickle feature from higher resolution VLA observations (YZ86), and recent H78 $\alpha$ emission observations made at Greenbank (Anantharamaiah and Yusef-Zadeh, 1988) show that some of the more diffuse material surrounding the Radio Arc, and associated with the helical filaments, also has a velocity around $+20 \mathrm{~km} \mathrm{~s}^{-1}$. It should be noted here that this material at $20 \mathrm{~km} \mathrm{~s}^{-1}$ does not appear to be connected with the $20 \mathrm{~km} \mathrm{~s}^{-1}$ material seen in the SW of the Sgr A complex, which has been called the ' $20 \mathrm{~km} \mathrm{~s}^{-1}$ cloud' by various authors. Figure 3 also shows contours of $3 \mathrm{~cm}$ polarized continuum emission (Seiradakis et al. 1985) superposed upon the HI optical depth at $+20 \mathrm{~km} \mathrm{~s}^{-1}$. The $3 \mathrm{~cm}$ map shows three distinct regions of polarization; two of these are from large lobes past the $N$ and $S$ ends of the Arc, and the third, marked as source A, is from the central Arc just below G0.18-0.04. We see that the central region coincides almost exactly with the gap in the $20 \mathrm{~km} \mathrm{~s}^{-1} \mathrm{HI}$ optical depth. This therefore suggests a scenario in which the filaments of the Arc are entirely non-thermal in nature but suffer from the depolarization of most of their synchrotron emission by ionized material associated with the $+20 \mathrm{~km} \mathrm{~s}^{-1}$ gas. A natural consequence of this would then be that it is the $20 \mathrm{~km} \mathrm{~s}^{-1}$ gas which is intimately related to the Arc instead of, or as well as, the $40 \mathrm{~km} \mathrm{~s}^{-1}$ cloud. If one is to believe that the $\sim 20 \mathrm{~km} \mathrm{~s}^{-1}$ gas is closely associated with the Arc, it is obviously important to try to show that the material is not simply a line of sight feature. There are several arguments which point towards this cloud lying close to the galactic centre. Firstly, the formaldeyde absorption observations of Güsten and Downes (1980), revealed a cloud at around $+25 \mathrm{~km} \mathrm{~s}^{-1}$ arross the northern portion of the Radio Arc and coinciding with the upper section of the HI cloud at $20 \mathrm{~km} \mathrm{~s}^{-1}$. They estimate that this cloud is fairly dense, has a mass of $\sim 4 \times 10^{5} M_{\odot}$, and displays linewidths of $\sim 30 \mathrm{~km} \mathrm{~s}^{-1}$ - characteristics of galactic centre clouds rather than of normal disk clouds. From our HI observations we can put a crude lower limit on the mass of the cloud at $20 \mathrm{~km} \mathrm{~s}^{-1}$ across the northern section of the Arc; $M_{\text {cloud }} \gtrsim 6 \times 10^{4} M_{\odot}$. Secondly, Figure 4 shows a ${ }^{12} \mathrm{CO} \mathrm{J}=2 \rightarrow 1$ spectrum taken at $\alpha=17^{h} 43^{m} 9.6^{s}, \delta=-28^{\circ} 47^{\prime} 8.0^{\prime \prime}$, a point on the Arc where the HI optical depth at $\sim+20 \mathrm{~km} \mathrm{~s}^{-1}$ is large. The large linewidth of the $+20 \mathrm{~km} \mathrm{~s}^{-1}$ feature shown here $\left(\Delta v_{F W H M} \simeq 25 \mathrm{~km} \mathrm{~s}^{-1}\right)$ again suggests that the $20 \mathrm{~km} \mathrm{~s}^{-1}$ material lies in the vicinity of the galactic centre. However, more convincing 


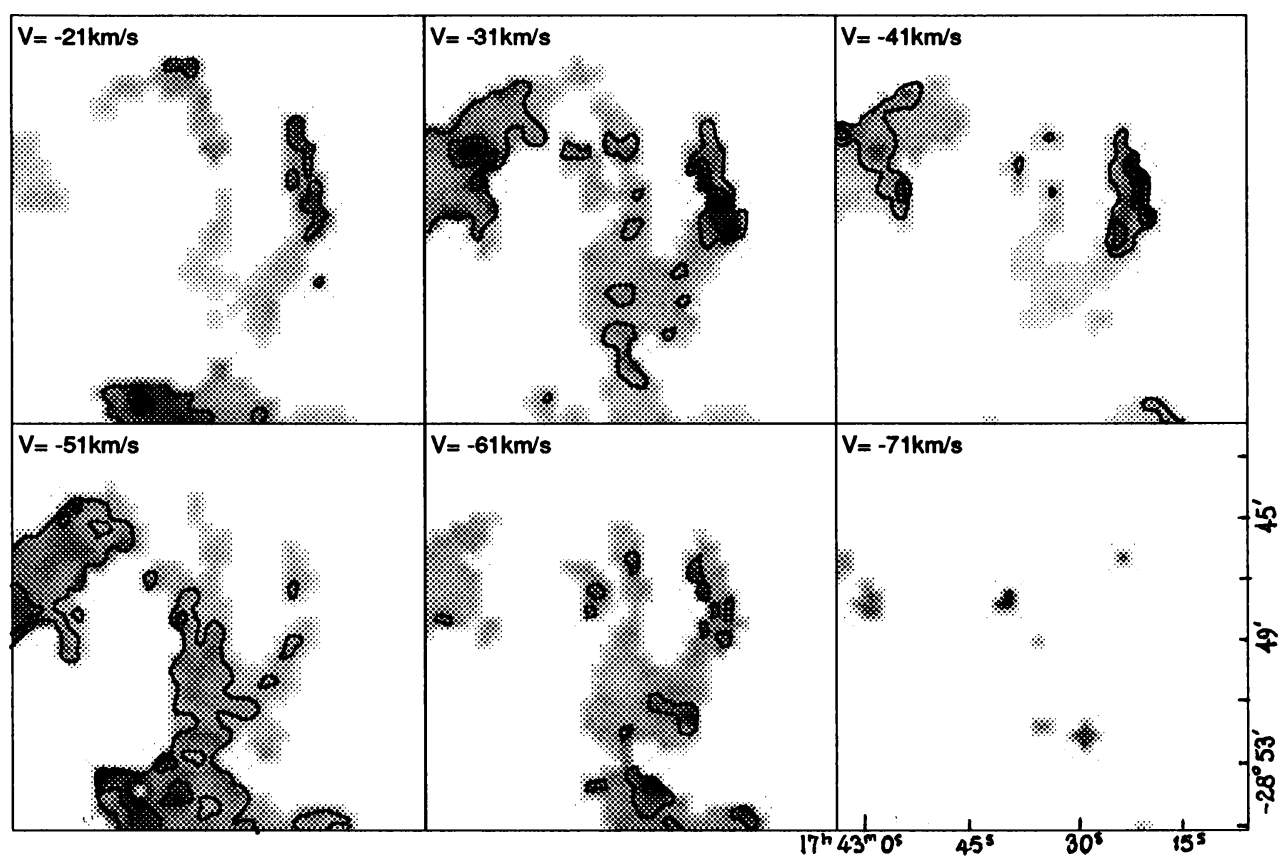

Figure 5: Maps of HI optical depth over the arched filaments for six negative velocity channels. In each map the scale is linear and the ranges of $\int_{\Delta v \sim 10} \tau d v$ are 0 to $1.3 \mathrm{~km} \mathrm{~s}^{-1}\left(-20 \mathrm{~km} \mathrm{~s}^{-1}\right.$ material), 0 to $1.7 \mathrm{~km} \mathrm{~s}^{-1}$ ( $-30 \mathrm{~km} \mathrm{~s}^{-1}$ material), 0 to $2.05 \mathrm{~km} \mathrm{~s}^{-1}$ (-40 $\mathrm{km} \mathrm{s}^{-1}$ material), 0 to $1.82 \mathrm{~km} \mathrm{~s}^{-1}$ $\left(-50 \mathrm{~km} \mathrm{~s}^{-1}\right.$ material), 0 to $1.20 \mathrm{~km} \mathrm{~s}^{-1}\left(-60 \mathrm{~km} \mathrm{~s}^{-1}\right.$ material $)$ and 0 to $0.37 \mathrm{~km} \mathrm{~s}^{-1}\left(-70 \mathrm{~km} \mathrm{~s}^{-1}\right.$ material). In each frame contours of $\int_{\Delta v \simeq 10} \tau d v=0.5$ and 1.0 are marked.

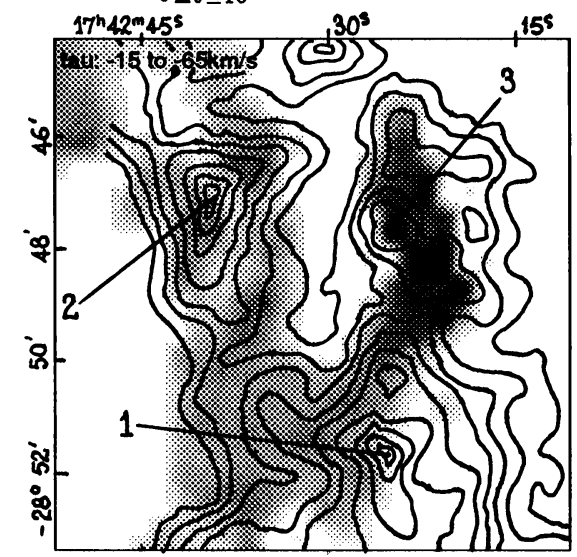

Figure 6: Contours of CS $\mathrm{J}=2 \rightarrow 1$ emission in the range +5 to $-55 \mathrm{~km} \mathrm{~s}^{-1}$ (taken from Serabyn and Güsten, 1987) across the arched filaments, overlayed onto a greyscale of HI optical depth integrated over a $50 \mathrm{~km} \mathrm{~s}^{-1}$ velocity interval between -15 and $-65 \mathrm{~km} \mathrm{~s}^{-1}\left(\int_{-65}^{-15} \tau(\mathrm{HI}) d v\right)$. The optical depth map scale is linear and ranges from 0 to $4.21 \mathrm{~km} \mathrm{~s}^{-1}$. The CS contour levels are $10,14,18,22,30,40,50,60,70 \mathrm{~K} \mathrm{~km} \mathrm{~s}^{-1}$. 
evidence would be spectra from a molecule such as CS which traces dense gas and is less likely to have a line of sight origin. Thus, the various evidence suggests that the $40 \mathrm{~km} \mathrm{~s}^{-1}$ cloud and the $20 \mathrm{~km} \mathrm{~s}^{-1}$ material both show signs of a physical association with the Radio Arc. While, on the basis of the HI absorption data, we would tend to favour an interaction between the Arc and the $20 \mathrm{~km} \mathrm{~s}^{-1}$ gas, the issue remains essentially open, and there is an obvious need for high resolution molecular observations to shed new light on the matter.

-10 to $-60 \mathrm{kms}^{-1}$ Material: The Arched Filaments: The arched filaments, or 'Arches', occurring at $\alpha \sim 17^{h} 42^{m} 30^{s}$ and $\delta \sim-28^{\circ} 48^{\prime}$, appear to be predominantly thermal structures. High resolution CS $\mathrm{J}=2 \rightarrow 1$ mapping of the Arches region (Serabyn \& Güsten, 1987 [hereafter, SG87]) shows clearly that the bulk of the molecular gas appears to be both spatially and kinematically associated with the ionized filaments. The HI optical depth maps of the Arches for velocity channels centred on -21 to $-62 \mathrm{~km} \mathrm{~s}^{-1}$, as seen in Figure 5 , show an overall correlation in velocity with the kinematic structure observed in the molecular and ionized gas (SG87, YZ86). The velocity distribution of the HI gas in front of the Arches is such that the more negative velocity gas is observed closest to $\mathrm{Sgr} \mathrm{A}$, with the velocity becoming more positive as we move northwards along the filaments towards the Radio Arc. Such a velocity gradient is seen in the CS observations (SG87) and, remarkably, also in the ionized gas distribution (YZ86). Thus, we conclude that the HI gas is closely associated with the molecular and ionized materials. Figure 6 shows the map of CS $\mathrm{J}=2 \rightarrow 1$ emission (SG87) integrated between 5 and $-55 \mathrm{~km} \mathrm{~s}^{-1}$ overlayed onto a greyscale of $\tau(\mathrm{HI})$ integrated over 5 velocity channels giving an approximate range of -15 to $-65 \mathrm{~km} \mathrm{~s}^{-1}$. Peaks 1 and 2 in the CS distribution (as referred to by SG87) are marked on the map, and a further, weaker peak (peak 3) is also marked. From the CS data we have estimates of the $\mathrm{H}_{2}$ column densities at these peaks: Peak $1, \mathrm{~N}\left(\mathrm{H}_{2}\right) \simeq(26 \pm 13) \times 10^{22} \mathrm{~cm}^{-2}$; Peak 2, $\mathrm{N}\left(\mathrm{H}_{2}\right) \simeq(17 \pm 8) \times 10^{22} \mathrm{~cm}^{-2} ;$ Peak $3, \mathrm{~N}\left(\mathrm{H}_{2}\right) \sim 8.2 \times 10^{22} \mathrm{~cm}^{-2}$. It is instructive to compare these values with those estimated from the HI data: Peak $1, \mathrm{~N}\left(\mathrm{H}_{2}\right) \lesssim 4.2 \times 10^{22} \mathrm{~cm}^{-2}$; Peak 2, $\mathrm{N}\left(\mathrm{H}_{2}\right) \lesssim 4.2 \times 10^{22} \mathrm{~cm}^{-2} ;$ Peak $3, \mathrm{~N}\left(\mathrm{H}_{2}\right) \lesssim 8.8 \times 10^{22} \mathrm{~cm}^{-2}$ [over the velocity interval -15 to $-65 \mathrm{~km} \mathrm{~s}^{-1}$ multiplied by a factor of $6 / 5$ in order to make the intervals comparable. Note that these estimates rely on various approximations $\left(T_{\text {spin }}\right.$ and $\mathrm{HI}: \mathrm{H}_{2}$ ratio $)$ and therefore contain a considerable degree of uncertainty]. Thus, we see that while the column densities over peak 3 are in fairly good agreement, over peaks 1 and 2 the $\mathrm{N}\left(\mathrm{H}_{2}\right)$ arrived at from the HI observations are roughly a factor of 6 or so lower than those calculated from the CS data. One is thus led to believe that around peaks 1 and 2 much of the CS molecular gas lies behind the ionized filaments and is therefore not accounted for in the calculations made using the HI optical depth maps. Another aspect of the HI absorption seen at $\sim-30 \mathrm{~km} \mathrm{~s}^{-1}$ is the appearance of the northern section of the Radio Arc and sickle feature, G0.18-0.04. Absorption of gas at these velocities against the Arc shows clearly that this ' $-30 \mathrm{~km} \mathrm{~s}^{-1}$ cloud' is more extended than was known previously. There does appear to be evidence for an anticorrelation of the $\mathrm{HI}$ gas with the radio continuum, particularly to the immediate north of the sickle. It may therefore be plausible to suggest a geometry in which the Arches and the $-30 \mathrm{~km} \mathrm{~s}^{-1}$ material are associated with each other and also with the non-thermal filaments in the continuum Arc, thus adding more weight to the arguments for the Arc and Arches being parts of a single, connected structure.

Expanding Molecular Ring Feature: Figure 1 shows that there is a patch of absorption 
over the southern tip of the Arc occurring at $\sim-130$ to $-140 \mathrm{~km} \mathrm{~s}^{-1}$. This absorbing gas is from the feature thought to be the front section of an expanding molecular ring at $R \sim 200 \mathrm{pc}$ (see Bania, 1977). Since we observe no absorption against the northern Arc by the rear section of this ring $\left(\sim+170 \mathrm{~km} \mathrm{~s}^{-1}\right)$, it can thus be inferred that the Radio Arc does indeed lie within the ring, i.e. within $\sim 200 \mathrm{pc}$ of the galactic centre. This was also the conclusion reached by Güsten and Downes, 1980 , from their $\mathrm{H}_{2} \mathrm{CO}$ absorption observations of the region.

\section{References}

Anantharamaiah, K. \& Yusef-Zadeh, F. 1988 presented at the

IAU Symposium No.136 on the Galactic Center, Ed. M. Morris.

Bally, J., Stark, A. A., Wilson, R. W. \& Henkel, C. 1987, Ap. J. Suppl., 65, 13.

Bania, T. M. 1977. Ap. J., 216, 381.

Güsten, R. \& Downes, D. 1980, Astr. Ap., 87, 6.

Güsten, R., Walmsley, C.M. \& Pauls, T. 1981, Astr. Ap., 103, 197.

Lasenby J., Lasenby, A.N. and Yusef-Zadeh, F. 1988, submitted to Ap.J.

Liszt, H. S., Burton, W. B. \& van der Hulst, J. M. 1985, Astr. Ap., $142,237$.

Pauls, T. \& Mezger, P.G. 1980, Astr. Ap., 85, 26.

Seiradakis, J. H., Lasenby, A. N., Yusef-Zadeh, F., Wielebinski, R. \& Klein, U. 1985, Nature, 317, 697.

Serabyn E. \& Güsten, R. 1987, Astr. Ap., 184, 133. [SG87]

Yusef-Zadeh, F. 1986, Ph.D Thesis, Columbia University. [YZ86]

Yusef-Zadeh, F., Morris, M. \& Chance, D. 1984, Nature, 310, 557.

Yusef-Zadeh, F., Morris, M. \& van Gorkom, J.H. 1987, in Proc. of the Symposium on the Galactic Center in Honor of C.H. Townes, Ed. D.C. Backer (New York, AIP), p190. 